\title{
Zwischen peace line und green line: Belfast und Beirut im zeitgenössischen Film
}

Als geteilte Städte sind Belfast und Beirut zur Inspirationsquelle vieler Drehbuchautoren und Regisseure geworden. In ihrem jeweiligen segmentierten Stadtgebiet spiegelt sich die Struktur einer Gesellschaft wider, die durch einen ethno-religiösen Konflikt in zwei feindliche Lager gespalten wurde. Während sich im Nordirland-Konflikt Katholiken und Protestanten gegenüberstanden, bekämpften sich im libanesischen Bürgerkrieg Christen und Muslime. Im Kontext des Konzepts ,Paragesellschaften“ stellen Nordirland und der Libanon jedoch Sonderfälle dar. Aufgrund einer internen Spaltung teilten sich die nordirische und die libanesische Gesellschaft in zwei ,Paragesellschaften“, die im Sinne zweier einander feindlich gestimmter Kulturgemeinschaften aufzufassen sind. Als Zentrum des jeweiligen Konfliktes zeigen sich die Konsequenzen der ethnoreligiösen Spannungen in Beirut und Belfast deutlicher als im Rest des Landes bzw. der Region. Sowohl in Belfast als auch in Beirut grenzten sich beide ,Paragesellschaften' nicht nur territorial voneinander ab, sondern entwickelten ebenfalls unterschiedliche Denkweisen sowie parallel existierende gesellschaftliche Strukturen.

Die territoriale und gesellschaftliche Teilung Belfasts und Beiruts ist ein beliebtes Thema im zeitgenössischen Film, international bekannte Filme sind dabei unter anderem Jim Sheridans The Boxer (1997), Yann Demanges '71 (2014) sowie Ziad Doueris West Beirut (1998) und ZoZo (2005) von Josef Fares. In filmischen Darstellungen beider Städte wird dabei nicht nur mit physischen Absperrungen und Barrikaden gespielt, sondern auch mit den zwischen den beiden ,Paragesellschaften' existierenden psychologischen Barrieren. Um die unterschiedlichen Verfilmungen der beiden Metropolen zu verstehen, ist eine kurze Einführung in ihre Stadtgeschichte bzw. die Entwicklung des jeweiligen Konflikts erforderlich.

\section{Belfast und der Nordirland-Konflikt}

Bis heute ist Belfasts urbane Topographie von einer territorialen Fragmentierung in katholische und protestantische Territorien gezeichnet, die insbesondere die von der Arbeiterklasse bewohnten Stadtteile prägt. Von 51 Stadtbezirken beste-

Ә Open Access. (C) 2021 Stephanie Schwerter, publiziert von De Gruyter. (cc) BY-NC-ND Dieses Werk ist lizenziert unter einer Creative Commons Namensnennung - Nicht-kommerziell - Keine Bearbeitung 4.0 International Lizenz. https://doi.org/10.1515/9783110707489-006 
hen 35 bis zu 95 Prozent aus Protestanten bzw. Katholiken. Aufgrund von administrativen Einrichtungen und Kaufhäusern stellt die Stadtmitte ein neutrales Territorium dar („Visualising the Conflict“). Der Osten Belfasts wird bis zu 88 Prozent von der protestantischen Bevölkerung bewohnt und umfasst lediglich eine kleine katholische Enklave namens Short Strand (Johnston 2008, 99). West-Belfast dagegen besteht aus einem ausgeweiteten katholischen Territorium, das die protestantische Enklave The Shankill umschließen. Während der Norden der Stadt ein patchwork aus aneinandergrenzenden Vierteln beider Kulturgemeinschaften darstellt, befindet sich im Süden Belfasts ein relativ überschaubares Gebiet in dem Angehörige der protestantischen und katholischen Mittelschicht friedlich zusammenleben (Heatly 2004, 61).

In Belfast visualisiert sich die städtische Spaltung durch eine Reihe sogenannter „boundary markers“ (O’Reilly 1998, 51), visueller Zeichen, die auf die Grenzen der einzelnen Territorien hinweisen. Eines dieser Phänomene ist die Bemalung von Bürgersteigkanten in den Farben des britischen Union Jack bzw. der irischen Trikolore (Smithey 2009, 85-106). Weitere Beispiele sind die unzähligen Flaggen, politischen Graffitis, Slogans und Akronyme sowie mehr als 300 murals, das heißt politischen Wandbemalungen, deren Thematik von historischen Ereignissen über die Glorifizierung lokaler Helden zu Machtgebärden paramilitärischer Organisationen reicht (Brothwick 2015, 6).

Die markantesten urbanen Barrieren sind jedoch die 97 peace lines, sprich Mauern, die gegnerische Territorien voneinander trennen („Belfast Interface Project“). Die an einigen Stellen bis zu sechs Meter hohen Betonabgrenzungen können mit einem Aufbau aus Stahl und Stacheldraht auf bis zu zwölf Meter erhöht werden (Feeney 2004, 25). Die ersten peace lines wurden von britischen Soldaten im Jahre 1969 konstruiert, um Katholiken und Protestanten davon abzuhalten, sich gegenseitig zu bekämpfen. Diese anfänglich als Provisorium geplanten Barrieren aus Holz und Stacheldraht entwickelten sich über die Jahre hinweg zu massiven Trennwänden. Die größte Anzahl von peace lines befindet sich in Nordund West-Belfast (Heatly 2004, 64). Die gesellschaftliche Spaltung der Region wird unter anderem durch ein konfessionell getrenntes Schulsystem verschärft, bei dem lediglich fünf Prozent der Schulen sowohl protestantische als auch katholische Kinder bzw. Jugendliche aufnehmen (Mitchell 2006, 60).

Die Wurzeln des nordirischen Konflikts liegen in der Kolonisierung Irlands im 16. Jahrhundert. Protestantische aus Nord-England und Schottland stammende Kolonisatoren siedelten sich im Norden der Insel an und bewegten sich teilweise weiter in den Süden (Ross 2005, 138-163). Daraus folgt, dass auch heute noch die meisten in Nordirland lebenden Protestanten Nachfahren schottischer bzw. englischer Siedler sind, während die nordirischen Katholiken von den Kelten abstammen, so dass die beiden ,Paragesellschaften` 
im Sinne zweier Ethnien aufzufassen sind. Katholiken sehen sich in der Regel als irisch und nehmen politisch eine nationalistische Haltung ein, während Protestanten sich in den meisten Fällen als Briten auffassen und einen unionistischen Standpunkt vertreten.

Als es 1948 zur Gründung der irischen Republik kam, wurde wegen der hohen Anzahl von Protestanten im Norden der nördliche Teil der Insel dem Vereinigten Königreich überlassen (Ross 2005, 46). Durch die wachsende Diskriminierung der katholischen Bevölkerung in Bezug auf Arbeitsplätze, Wahlrecht und Sozialwohnungen kam es im Jahre 1968 zum Ausbruch des NordirlandKonflikts, der im englischen Sprachraum weitgehend als The Troubles bezeichnet wird (MacKittrick und McVea 2001, 11-12). Aufgrund der gespannten Situation in Nordirland entstanden paramilitärische Organisationen auf beiden Seiten, von denen die IRA weltweit die bekannteste ist. ${ }^{1}$ Die gewaltsamen Auseinandersetzungen zwischen Protestanten und Katholiken forderten um die 4000 Todesopfer (Hanna 1999, 197). Politiker und Wissenschaftler sind jedoch bis heute uneins über das Ende des Nordirland-Konflikts. Manche sehen das Ende der Auseinandersetzung in dem 1998 geschlossenen Good Friday Agreement (Welch 2000, 364), andere argumentieren, dass erst durch die Wiederherstellung eines gemeinsamen Parlaments im Jahre 2007 ein Schlussstrich unter die Konfrontationen gezogen wurde (Melaugh).

\section{Beirut im libanesischen Bürgerkrieg}

Wie im Falle Belfasts liegt Beiruts urbane Fragmentierung in der Geschichte des Landes begründet. Seit Beginn des 18. Jahrhunderts etablierten sich Christen im Osten der Stadt, während sich Muslime im Westen niederließen (Asmar 2006, 74). Zur Zeit des französischen Mandats (1920-1943) begann sich insbesondere die christliche Bevölkerung mit der französischen Sprache und Kultur zu identifizieren, wodurch die kulturellen Unterschiede zwischen Christen und Muslimen vergrößert wurden (Naeff 2018, 20). In den 1950er und 60er Jahren erlebte das Land das sogenannte ,goldene libanesische Zeitalter“, das von einer florierenden Wirtschaft und einer lebendigen Kulturszene geprägt war. Zu dieser Zeit lebten Christen und Muslime trotz ihrer kulturellen und religiösen Differenzen friedlich zusammen. Beirut wurde zum intellektuellen Zentrum der arabischen

1 Weitere paramilitärische Organisationen auf der katholischen Seite waren unter anderem CIRA (Continuity IRA), RIRA (Real IRA), DAAD (Direct Action Against Drugs) und INLA (Irish National Liberation Army). 
Welt und von vielen als Paris des Orients bezeichnet (Kassir 2003, 16-17). Durch die zunehmende Zahl der palästinensischen Flüchtlinge veränderte sich jedoch die Demographie des Landes, so dass sich eine muslimische Mehrheit in der Bevölkerung bildete (Calame und Charlesworth 2009, 44).

Der Zwischenfall, an dem sich der libanesische Bürgerkrieg entzündete, ereignete sich am 13. April 1975. An diesem Tag wurde auf Pierre Gemayel, dem Leiter der christlichen Partei der Phalangisten, ein Attentat verübt. Gemayel überlebte zwar, sein Leibwächter sowie drei weitere Personen wurden jedoch getötet. Die Phalangisten gingen davon aus, dass es sich bei den Tätern um radikalisierte Palästinenser handelte und schossen daher aus Rache auf einen Bus mit palästinensischen Zivilisten. Nach dem Angriff, bei dem 27 Personen getötet und mehr als 20 verwundet wurden, kam es in Beirut zum Ausbruch unkontrollierbarer Gewalt (Llewllyn 2010, 60).

Die politische Situation schlug sich schnell im Stadtbild nieder. In kürzester Zeit entwickelte sich eine befestigte Demarkationslinie zwischen Ost und West. Die sogenannte green line schnitt durch die Stadt, zertrennte Hauptverkehrsstraßen und brachte den zentralen Verkehrskontenpunkt an der Place des Martyrs zum Stillstand (Kathib 2008, 62). Die Demarkationslinie mutierte zu einem neun Kilometer langen und zwischen 18 und 90 Meter breiten Niemandsland. Von beiden Seiten war sie mit Barrikaden befestigt, für deren Errichtung Sandsäcke, Stacheldraht, ausgebrannte Fahrzeuge, Zementblöcke, Schutt sowie vom Hafen gestohlene Container benutzt wurden (Calame und Charleworth 2009, 38). Neben der Linie kam es in der gesamten Stadt zur Errichtung von bis zu drei Meter hohen, diverse Enklaven umzäunenden Barrikaden (Calame und Charleworth 2009, 38).

Während des Bürgerkrieges war das christliche Ost-Beirut rechtsorientiert und politisch gegen Palästina gerichtet, während das muslimische West-Beirut eine linksorientierte pro-palästinensische Haltung vertrat (Naeff 2018, 23). Ähnlich wie in Belfast wachten paramilitärische Organisation beider Lager über die internen urbanen Grenzen (Bollens 2012, 148). Die Bewegungsfreiheit der meisten Einwohner Beiruts war während des Bürgerkrieges auf das Gebiet ihrer eigenen Kulturgesellschaft beschränkt (Mermier 2015, 75-76). Der gewaltsame Konflikt forderte das Leben von ca. 150000 Zivilisten und machte mehr als 30 Prozent der libanesischen Bevölkerung zu Flüchtlingen (Asmar 2006, 62). Das Ende des Bürgerkrieges begann sich in den letzten Monaten des Jahres 1989 mit der Unterzeichnung eines Friedensabkommens zwischen Muslimen und Christen abzuzeichnen. 1991 wurde das Abkommen durch einen weiteren Friedensvertrag bestätigt (Asmar 2006, 62). 


\section{Filmische Darstellung von politischer Gewalt}

Aufgrund ihrer urbanen Teilung und gewaltgeladener Atmosphäre wurden die Städte Belfast und Beirut zum Schauplatz zahlreicher Filme. Die meisten in Belfast und Beirut spielenden Filme wurden nach Beginn der 1990er Jahre gedreht. Obwohl auch in den 80er Jahren einige wenige Filme produziert wurden, trug die friedlichere Atmosphäre in den beiden Städten in den 90er Jahren zu einem Boom in der Filmindustrie bei. Die Gründe für diese Entwicklung sind in beiden Ländern jedoch verschieden.

In Nordirland erklärte die IRA 1994 erstmalig einen Waffenstillstand. Dadurch wurde es möglich, direkt vor Ort zu filmen. Zuvor mussten die meisten Belfast-Filme aus Sicherheitsgründen in Dublin, Manchester oder London gedreht werden (Hill 2006, 213). Nach der Waffenstillstandserklärung der IRA begannen verschiedene staatliche Organisationen, Drehbuchautoren und Regisseuren finanzielle Hilfe anzubieten (Barton 2004, 162). Wie in Nordirland waren in den meisten Teilen des Libanons Dreharbeiten vor Ort während des Bürgerkrieges unmöglich (Khatib 2008, 23). Die gesellschaftliche Spaltung verhinderte das Florieren der Filmindustrie, da das im Osten Beiruts gelegene Viertel Hamra - das libanesische Kulturzentrum des Films - nach Errichtung der Demarkationslinie für die Bewohner des Westens der Stadt nicht mehr zugänglich war (Khatib 2008, 25). Nach Ende des Konflikts wendeten sich zahlreiche libanesische Filmemacher an internationale Organisation, um ihre Projekte $\mathrm{zu}$ finanzieren, da von der libanesischen Regierung keine Unterstützung zu erwarten war (Armes 2015, 20).

Die meisten Filme, die sich mit dem nordirischen Konflikt bzw. dem libanesischen Bürgerkrieg beschäftigen, thematisieren nicht nur die territoriale Segmentierung der Stadt, sondern ebenfalls die Spaltung der Gesellschaft. Regisseure spielen dabei mit den inneren urbanen Grenzen, sowie der Präsenz der Armee, der Polizei und der diversen paramilitärischen Organisation. Barrikaden und Absperrungen sowie Schießereien und Bombenexplosionen bilden nicht selten den Hintergrund der Handlung. Das beliebteste Subgenre des nordirischen Films ist der sogenannte Troubles-Thriller. ${ }^{2}$ Diese Art von Thriller ist von einem realistischen, fast schon dokumentarischen Stil geprägt und handelt von paramilitärischen Gangs, die sich nicht nur gegenseitig bekämpfen, sondern auch unschuldige Zivilisten ins Visier nehmen. $\mathrm{Zu}$ dem traditionellen männlichen Figurenrepertoire des Troubles-Thriller zählen nach Bill Rolston und Gerry Smyth: der terrorist

2 Weitere Sub-Genres des nordirischen Films sind unter anderem die Komödie und die sogenannte Love-Across-the-Barricades Story bei der zwei Figuren der unterschiedlichen Kulturgemeinschaften durch ihren religiös-politischen Hintergrund davon abgehalten werden, zusammenzukommen. 
godfather - ein politisierter Kommandant -, der gunman - eine Art Handlanger der Obrigkeit - und der villain - ein unpolitisches Individuum, für das Gewalt lediglich eine Art von Spiel ist (Smith 1997, 114). Zu den weiblichen wiederkehrenden Figuren gehören die besorgte Mutter, die mörderische femme fatale, sowie das Äquivalent des männlichen villain in der Form einer dämonischen Frau (Rolston 1989, 44).

Ein weiteres Phänomen, das sowohl an in Belfast als auch in Beirut spielenden Filmen beobachtet werden kann, ist das Motiv des flâneurs. Ähnlich wie Baudelaires flâneur, der im 19. Jahrhundert durch Paris streunt und dabei die Stadt beobachtet und interpretiert $(1973,39)$, bewegen sich in Belfast und Beirut eine Reihe von Figuren durch die kriegsgezeichnete Stadt. Laut Richard Burton ist der flâneur als externer Beobachter zu sehen, der einerseits von der Menge losgelöst ist, andererseits aber mit der Stadt verschmilzt (1994, 2). Ein Belfaster bzw. Beiruter flâneur beobachtet dabei hauptsächlich die sich im Stadtbild widerspiegelnden Konsequenzen politischer Gewalt.

Auch wenn unter den libanesischen Filmen Vertreter des Thriller Genres zu finden sind, ist letzteres nicht als dominierende Form $\mathrm{zu}$ beobachten. Wie in Belfast-Filmen konzentrieren sich in Beirut spielende Filmen auf die gewaltsamen Auseinandersetzungen der beiden ,Paragesellschaften“. Die behandelte Themenpalette ist jedoch breiter. Neben den Aktionen paramilitärischer Organisationen spielen im libanesischen Film die Entführungen unbeteiligter Zivilisten eine wichtige Rolle sowie das Eintreiben von Schutzgeldern und die aus dem Süden des Landes nach Beirut kommenden Flüchtlinge. Soziale Unterschiede innerhalb der beiden Kulturgemeinschaften sind ebenfalls ein häufiges Thema. Die in Beirut spielenden Filme können jedoch nicht einem spezifischen Genre zugeordnet werden, da sie in ihrer Struktur und Filmweise voneinander abweichen.

Belfasts und Beiruts fragmentiertes Stadtbild steht im Zentrum der meisten Filme. Es geht oft darum, wie die Figuren das Territorium ihrer eigenen Kultgemeinschaft im Gegensatz zu feindlichen Sektoren auffassen. Durch die kognitiven Stadtpläne der Protagonisten wird dem Filmpublikum eine individuelle Perspektive der betreffenden Stadt vermittelt. Das Konzept der kognitiven Geographie, der mental maps, wurde von dem Soziologen Kevin Lynch in seinem Buch The Image of the City (1960) entwickelt. Es handelt sich dabei um kognitive Stadtpläne, die sich durch die Konzentration auf bestimmte Aspekte der urbanen Landschaft in der Vorstellung des Beobachters bilden (Lynch 1960, 1-4). Laut Mark Gottdiener verfügt jede soziale Gruppe ihre eigene Auffassung einer bestimmten Stadtgeographie (1986, 206). Liam Kennedy erklärt, dass ein Ort für jeden Stadtbewohner mit spezifischen persönlichen Geschichten, Emotionen, Bildern oder Erinnerungen verbunden ist und so auf jedem kognitiven Stadtplan 
eine andere Form annimmt (2003, 7). In Beirut- und Belfast-Filmen orientieren sich die Figuren an ihren individuellen mental maps, um sich gefahrlos durch die verschiedenen Territorien der Stadt bewegen zu können.

\section{Sackgassen und Irrwege: Belfasts territorialen Grenzen}

Thaddeaus O'Sullivans Grenzenloser Haß [Nothing Personal] (1995) und Marc Evans Resurrection Man (1999) sind Beispiele des traditionellen nordirischen Troubles-Thrillers. Beide Filme beschäftigen sich mit der Shankill-Butcher-Gang, einer besonders grausamen protestantischen paramilitärischen Organisation, die zwischen 1975 und 1985 in West-Belfast zahlreiche katholische Zivilisten zunächst folterte und dann ermordete (Dillon 1990). Der Realität entnommene Themen, die in Zusammenhang mit dem nordirischen Konflikt stehen, sind charakteristisch für den Troubles-Thriller. In dieser Art von Film kommt es häufig zu einer einseitigen Abbildung der Belfaster Gesellschaft, da die Handlung stets aus dem Blickwinkel einer Kulturgemeinschaft dargestellt wird. Die Stadt Belfast wird generell als Moloch gezeigt, aus dem es zu entkommen gilt.

Der Protagonist in Grenzenloser Haß ist der Katholik Liam, ein alleinstehender Vater zweier Kinder. Der Film beginnt mit der Explosion einer von der IRA gelegten Bombe. In der ersten Szene steigt Liam über die Überreste zerstörter Häuser und hilft der britischen Armee, Verwundete und Tote zu bergen, so dass schon zu Anfang des Films ein grausames Bild der Stadt gezeichnet wird. Die Handlung dreht sich um die Vergeltungsschläge der $U V F$, dem protestantischen Äquivalent der IRA. In O'Sullivans Thriller wird Belfast als ein undurchdringliches Labyrinth von katholischen und protestantischen Territorien dargestellt. Die innerstädtischen Grenzen werden dabei deutlich mit Wandgemälde, Barrikaden und Flaggen visualisiert. Die Handlung spielt nachts in den Hinterzimmern dunkler Kneipen sowie in leeren Fabrikhallen und auf verlassenen Parkplätzen. Die finstere Atmosphäre wird durch Nebel und die Wahl eines gedämpften bläulichen Lichts unterstützt. Der ständige Lärm von über der Stadt fliegenden Armee Hubschraubern verstärkt dabei den bedrohlichen Ton des Films.

Die Atmosphäre in Resurrection Man ist eine ähnliche. Eine dunkle Beleuchtung, die laut Evans dem amerikanischen Film noir nachempfunden ist (MacNamee 1998, 14), signalisiert Bedrohung und Gefahr. Wie in Grenzenloser Haß prägen Hubschrauber der britischen Armee, Barrikaden sowie diverse boundary markers das Belfaster Stadtbild. Zahlreiche Szenen tragen sich ebenfalls nachts zu. Der Protagonist des Films ist Victor Kelly, eine Figur, die dem Anführer der Shankill-Butcher- 
Gang nachempfunden ist. Im Laufe des Films entwickelt sich Victor vom gunman zum terrorist godfather. Er begeht immer grausamere Morde an Katholiken und wird nach und nach zum geführchteten Herrscher über West-Belfast. Während Nothing Personal in den Vierteln beider Kulturgemeinschaften spielt, konzentriert sich die Handlung in Resurrection Man auf die protestantische West-Belfaster Enklave The Shankill.

In beiden Filmen sind die mental maps der Protagonisten von Bedeutung. Liam und Victor besitzen präzise kognitive Stadtpläne, auf denen die Grenzen zwischen katholischen und protestantischen Vierteln abgebildet sind. Sobald die Figuren ihre Orientierung verlieren und unbewusst auf feindliches Territorium geraten, bringen sie sich in Gefahr. In Grenzenloser Haß setzt der verwundete Protagonist nach einem nächtlichen Straßenkampf unabsichtlich Fuß auf protestantisches Gebiet. Da die geographischen Details des feindlichen Territoriums auf seiner persönlichen mental map nicht existieren, gerät er immer tiefer ins Labyrinth der ihm unbekannten Straßen. Die alleinstehende protestantische Mutter Ann beobachtet den blutüberströmt umherirrenden Mann von ihrem Fenster aus und bietet ihm aus Mitleid an hereinzukommen. Als sie sich um seine Wunden kümmert, entsteht zwischen den beiden Figuren eine Unterhaltung, die ein gegenseitiges Interesse andeutet. Liam und Ann sind sich jedoch bewusst, dass aufgrund ihrer Zugehörigkeit zu zwei verfeindeten ,Paragesellschaften' eine potentielle Liebesbeziehung für sie und ihre Familien zu gefährlich wäre und daher nicht in Frage kommt. Als Liam Anns Haus verlässt, wird er auf protestantischem Territorium von UVF-Mitgliedern bedroht und kommt nur knapp mit dem Leben davon. Erst als er den katholischen Teil Belfasts erreicht, kann er sich wieder in Sicherheit fühlen.

Wie in Grenzenloser Haß helfen auch in Resurrection Man mental maps dem Protagonisten dabei, sich im Netz der Belfaster Straßen zurechtzufinden. In mehreren Szenen wird Viktor von seinen Untergebenen mit dem Auto durch die Stadt gefahren, wobei er mit geschlossenen Augen die Namen der Straßen nennt, auf denen sie sich gerade befinden. Viktors Macht über die protestantische Shankill-Enklave wird durch seine Beherrschung der örtlichen Geographie verdeutlicht. Der kognitive Stadtplan des Protagonisten ist so ausgeprägt, dass es Viktor sogar möglich ist, anhand von Gerüchen bestimmte Orte zu bestimmen. Viktors Streifzüge durch das nächtliche Belfast erinnern an die Spaziergänge der flâneur-Figur Baudelaires (Benjamin 1973, 39). Ähnlich wie der Pariser flâneur beobachtet und interpretiert Viktor sein urbanes Umfeld. Auf Viktors mental map ist Belfast in katholische und protestantische Territorien eingeteilt, die wiederum mit bestimmten Verbrechen verbunden sind. Mit Hilfe seines kognitiven Stadtplans lauert Viktor neuen Opfern auf, die er aufgrund ihrer Bewegung im städtischen Raum als Katholiken identifiziert. Nach Charles Armstrong wird 
Viktor so zum negativen Spiegelbild eines Sherlock Holmes (2018, 70). Während der englische Detektiv dank seiner mental maps im viktorianischen London Verbrechen auf die Spur kommt, benutzt Viktor seine Kenntnis des Belfaster Stadtbilds, um seine eigenen Morde zu planen $(2018,70)$. Viktors schwindende Beherrschung der Belfaster Geographie spiegelt seinen psychologischen Zustand wider, da durch einen zunehmenden Drogenkonsum sein internes Navigationssystem aus dem Gleichgewicht gebracht wird. Die an seine Gangster-Kollegen gerichteten Ortsangaben werden immer unzuverlässiger, so dass eine nächtliche Autofahrt nicht selten in einer Sackgasse endet. Viktors Kontrollverlust über sein Territorium symbolisiert dabei auch den graduellen Verlust seiner Macht.

\section{Beirut zwischen Ost und West}

Wie in Nothing Personal und Resurrection Man steht in den folgenden libanesischen Filmen die Fragmentierung des Stadtgebiets im Mittelpunkt. Im Schatten der Stadt [Tayf al-Madina] von Jean Chamoun (2000) und Bahij Hojeijs Film Feuerring [Zinnar al-Nar] (2003) spielen während des libanesischen Bürgerkriegs und stellen Beirut als zweigeteilte Stadt dar. Auch wenn in Feuerring und Im Schatten der Stadt die gewaltsamen Auseinandersetzungen der beiden ,Paragesellschaften' die Handlung prägen, sind die beiden Filme nicht im Thriller-Stil gedreht. Im Gegensatz zu Nothing Personal und Resurrection Man zielen sie weniger auf Spannung ab und sind in einem wesentlich langsameren Tempo gefilmt.

Der Protagonist in Chamouns Film ist Rami, ein 12-jähriger muslimischer Junge, der vor Ausbruch des Bürgerkrieges mit seiner Familie vor den israelischen Angriffen im Süden des Landes in die Hauptstadt flieht. In Beirut angekommen freundet sich Rami mit den Kindern aus der Nachbarschaft an und verliebt sich in Yasmin, ein christliches Mädchen aus einer gutbetuchten Familie. Vor Beginn der gewaltsamen Auseinandersetzungen spielen religiöse und soziale Unterschiede keine Rolle. Die Familien der beiden Kinder leben friedlich im selben Stadtviertel in West-Beirut und pflegen ein freundschaftliches Verhältnis. Die politische Situation in der Stadt wird jedoch zunehmend angespannt und paramilitärische Organisationen beider Seiten beginnen die Straßen zu übernehmen. Nach Ausbruch des Bürgerkrieges verlässt Yasmins Familie den Osten der Stadt, um im christlichen West-Beirut Zuflucht zu finden. Durch die physische Abriegelung von Ost und West verliert Rami den Kontakt zu Yasmin, hofft jedoch, sie eines Tages wiederzufinden. Die zunehmende Spaltung der Stadt wird mit dem Erwachsenwerden des Protagonisten parallelisiert. Nach der Entführung seines Vaters tritt der friedliebende Rami einer muslimischen paramilitärischen 
Organisation bei, um seine Kulturgemeinschaft zu beschützen. Die Stadt zerfällt zusehends in zwei ethno-religiöse Territorien, die von sich feindlich gegenüberstehenden ,Paragesellschaften‘ beherrscht werden.

In Hojeijs im Jahre 1985 spielenden Feuerring wird Beirut schon zu Beginn als gespaltene Metropole dargestellt, in der Ruinen und Schutt das Stadtbild prägen. Der Protagonist des Films ist der Französischprofessor Chafik, der nach einem längeren Auslandsaufenthalt in die Stadt zurückkehrt. Anders als in Im Schatten der Stadt ist jedoch unklar, welcher Kulturgemeinschaft er angehört, und in welchen Vierteln sich die Handlung abspielt. Hojeij erklärt, er habe die Figur absichtlich abstrakt gehalten, um ihr keine bestimmte religiös-politische Identität geben $\mathrm{zu}$ müssen (Khatib 2008, 173). Im Verlauf des Films dringt der Bürgerkrieg zunehmend in Chafiks Leben ein und seine Vorlesungen werden regelmäßig von Bombenexplosionen gestört. Die Schlüsselszene des Films spielt im Keller der Universität, in dem der Protagonist mit seinen Studenten Zuflucht sucht, um sich vor einem Bombenangriff zu schützen. In der Dunkelheit klammert sich eine verschreckte Studentin an Chafik und küsst ihn, was den introvertierten, alleinstehenden Protagonisten gänzlich aus dem Gleichgewicht bringt. Im allgemeinen Chaos verschwindet sie jedoch nach Ende des Angriffs, so dass er nicht weiß, um welches Mädchen es sich gehandelt hat. Im weiteren Verlauf des Filmes träumt Chafik von der mysteriösen jungen Frau und versucht fortan sie wiederzufinden.

In Im Schatten der Stadt und Feuerring fungieren beide Protagonisten als Reflektorfiguren, durch deren Augen die Verwüstung der Stadt abgebildet wird. In Form von Beiruter flâneuren streifen Rami und Chafic durch die verschiedenen Viertel und beobachten die sich im Stadtbild widerspiegelnde politische Gewalt. Als Krankenwagenfahrer bewegt sich Rami durch das zerstörte Beirut und betrachtet mit Schrecken die Ruinen. Das Stadtgebiet steht gänzlich unter Kontrolle der paramilitärischen Organisationen beider Seiten, so dass es für die Beiruter Bewohner unmöglich ist, zur anderen Seite der Stadt zu gelangen, ohne ihr Leben zu riskieren. Aufgrund seiner kognitiven Stadtgeographie weiß Rami, auf welchen Territorien er sich um welche Verletzten kümmern darf, ohne als Verräter seiner kulturellen Gruppierung zu gelten. Der Film endet mit einer Sequenz, in der Beirut nach Ende des Bürgerkriegs gezeigt wird. Obwohl die Häuser und Straßen noch in Trümmern liegen, sind die Grenzen der Stadt erneut geöffnet. Geschäftsmänner und Immobilienhändler strömen herbei, um finanziell vom Wiederaufbau der Stadt zu profitieren. Auch wenn die Atmosphäre in der Stadt sich zum Positiven wandelt, nimmt der Film für Rami ein tragisches Ende. Der Protagonist muss erfahren, dass Yasmin, auf die er während des gesamten Bürgerkrieges gewartet hat, glücklich verheiratet ist und eine Tochter hat. Der Film suggeriert auf diese Weise, dass die territoriale und gesellschaftliche Spaltung 
der Stadt den Protagonisten davon abgehalten hat, mit seiner Jugendliebe zusammenzukommen.

Auch in Hojeijs Feuerring ist es dem Protagonisten unmöglich, das Mädchen wiederzufinden, in das er sich im Dunkeln des Universitätskellers verliebt hat. In seinem Wagen fährt Chafik durch das nächtliche Beirut und träumt von der Studentin. Wie ein von der Menge losgelöster flâneur betrachtet er die in Schutt und Asche liegende Stadt. Chafiks melancholische Stimme aus dem Off kommentiert die Szene wie folgt:

Wären die Straßen sicher, würde ich die ganze Nacht durchwandern. Wäre ich ein Dich-
ter, würde ich die schönsten Gedichte schreiben. Wo ist sie wohl jetzt? Was trägt sie? Wel-
cher Rock bedeckt ihre Beine? Welche Bluse ist um ihre Brust gebunden? Welcher Gürtel
umschlingt ihre Taille? Was hält sie ab davon zu mir zu kommen? Welche Barrikade? Wel-
cher Grund? Könnte sie nur ein Traum sein, der zu Ende gegangen ist?

(Hojeij 2003, 00:26.16-00:26:49)

Dank seiner mental map ist sich der Protagonist dessen bewusst, dass die Straßen der Stadt kein sicheres Territorium darstellen. Er fragt sich, welche „Barrikade“ das Mädchen davon abhält, $\mathrm{zu}$ ihm zu kommen und spielt so auf die verschiedenen physischen, religiös-politischen bzw. sozialen Hindernisse an, die zwischen ihnen stehen. Im geteilten Beirut verweist das Wort ,Barrikade“ zunächst auf die den Osten und Westen der Stadt voneinander trennende Demarkationslinie. Dies bedeutet, dass Chafik und die Studentin potentiell von physischen Grenzen voneinander ferngehalten werden. Im weiteren Sinne spielt der Terminus jedoch auf die religiös-politischen Barrieren zwischen den beiden ,Paragesellschaften' an und impliziert so, dass die junge Frau der entgegengesetzten Kulturgemeinschaft angehört. Ein weiteres Hindernis zwischen den beiden Figuren ist unter Umständen ihr Abhängigkeitsverhältnis, das eine Beziehung zwischen Professor und Studentin verbietet.

Realität und Traum verschmelzen in Chafiks Vorstellung miteinander, und plötzlich erscheint das Mädchen wie aus dem Nichts in seinem Wagen. Am nächsten Kontrollpunkt werden beide Figuren jedoch von den örtlichen paramilitärischen Kämpfern angehalten. Während Chafik unter dem Vorwand festgenommen wird, einen gestohlenen Wagen zu fahren, schicken die Milizia-Männer das Mädchen nach Hause. Er ist gezwungen, die Nacht am Kontrollpunkt zu verbringen, bevor er am nächsten Morgen freigelassen wird. Die Filmzuschauer zu verstehen jedoch erst später, dass die Episode gänzlich Chafiks Fantasie entsprungen ist. Im Laufe des Films vermag der Protagonist immer weniger zwischen Realität und Traum zu unterscheiden. Ähnlich wie Victor in Resurrection Man geht Chafiks Abgleiten in den Wahnsinn mit dem Verlust seiner kognitiven Geographie der Stadt einher. Als sich der Protagonist am nächsten Tag an den Ort begibt, an 
dem er vermeintlich eine Nacht lang festgehalten wurde, muss er feststellen, dass der Kontrollpunkt gar nicht existiert. Daraufhin scheint Chafik gänzlich den Verstand zu verlieren: Schreiend weigert er sich weiterzufahren, da es keinen Wächter gibt, der seinen Pass kontrolliert. Dieses Verhalten verdeutlicht, dass er nicht mehr im Stande ist, Beiruts innere Grenzen auf seinem kognitiven Stadtplan zu erkennen.

\section{Schlussfolgerung: Unterschiedliche Entwicklungen in der filmischen Darstellung der nordirischen und libanesischen ,Paragesellschaften ${ }^{6}$}

Wie die beispielhafte Analyse von Nothing Personal, Resurrection Man, Im Schatten der Stadt und Feuerring verdeutlicht, zeichnen sich in Belfast und Beirut spielende Filme durch eine Reihe thematischer und formaler Parallelen aus. Das fragmentierte Stadtbild, das von den Protagonisten durch ihre individuellen mental maps auf unterschiedliche Weise wahrgenommen wird, steht ebenso im Mittelpunkt der Handlung wie die zwischen den beiden ,Paragesellschaften“ existierenden physischen und psychologischen Barrieren. Auch ist der realistische, oft an Dokumentation grenzende Stil eine häufig zu beobachtende Gemeinsamkeit von Filmen, die sich mit dem Nordirland-Konflikt bzw. mit dem libanesischen Bürgerkrieg befassen.

Im filmischen Umgang mit dem jeweiligen Konflikt sind jedoch unterschiedliche Entwicklungen $\mathrm{zu}$ beobachten. Im nordirischen Film wird seit der Waffenstillstandserklärung der IRA im Jahre 1994 eine Tendenz zu einer humoristischen Darstellung des Nordirlandkonflikts deutlich. Aufgrund der beruhigten Lage begann eine junge Generation von Regisseuren und Drehbuchautoren, die Animositäten der beiden ,Paragesellschaften“ mit Ironie und Humor abzubilden. In komödienartigen Filmen werden die Anhänger paramilitärischer Organisationen als komische ungeschickte Gangster abgebildet sowie die Vertreter von Kirche und Staat verlacht. Ebenso werden die Gründe für den Konflikt ironisch beleuchtet und häufig als absurd dargestellt. Im Gegensatz dazu ist Humor seltener in libanesischen Filmen zu finden, die sich mit dem Bürgerkrieg auseinandersetzen. Dies mag einerseits daran liegen, dass aufgrund der instabilen politischen Situation im Mittleren Osten ein Ausbruch von Gewalt nicht auszuschließen ist, und daher die Bevölkerung des Landes noch keinen genügend großen mentalen Abstand zum Konflikt gefunden hat, um diesen mit Humor zu betrachten. Darüber 
hinaus ist es gesetzlich verboten, Filme $\mathrm{zu}$ drehen, welche bestimmte Bevölkerungsgruppen herabwürdigen oder beleidigen könnten („Intervention écrite de la sûreté générale“). Durch eine strenge Zensur werden Ironie und schwarzer Humor aus dem Kino verbannt, um so Spannungen zwischen den beiden ,Paragesellschaften' zu vermeiden. Im Falle des nordirischen Films ist abzuwarten, ob die post-Brexit-Situation die Auseinandersetzungen zwischen den beiden Kulturgemeinschaften erneut aufflammen lässt, und so Drehbuchautoren und Regisseure sich wieder von einer humoristischen Verarbeitung des Konflikts abwenden.

\section{Bibliographie}

Armes, Roy. New Voices in Arab Cinema. Bloomington: Indiana University Press, 2015.

Armstrong, Charles Ivan. „Violent Measures. Representation, Regulation and The Shankill

Butchers“. Mauern, Grenzen, Zonen. Geteilte Städte in Literatur und Film. Hg. Walburga

Hülk und Stephanie Schwerter. Heidelberg: Winter, 2018. 65-73.

Asmar, Fady. Atlas du Liban. Beirut: Presses de l'Université Saint-Joseph, 2006.

Barton, Ruth. Irish National Cinema. London: Routledge, 2004.

Benjamin, Walter. Charles Baudelaire. Ein Lyriker im Zeitalter des Hochkapitalismus. Frankfurt a.M.: Suhrkamp, 1974.

Bollens, Scott A. City and Soul in Divided Societies. New York: Routledge, 2012.

Brothwick, Stuart. The Writing on the Wall. A Visual History of Northern Ireland's Troubles. Liverpool: Bluecoat Press, 2015.

Burton, Richard. The Flâneur and His City. Patterns of Daily Life in Paris 1815-1851. Durham: University of Durham, 1994.

Calame, Jon und Esther Charleworth. Divided Cities. Belfast, Beirut, Jerusalem, Mostar, and Nicosia. Phidadelphia: University of Pennsylvania Press, 2009.

Dillon, Martin. The Shankill Butchers: A Case Study of Mass Murder. London: Arrow Books, 1990.

Executive Office (Northern Ireland): Together: Building a United Community (2013). https:// www.executiveoffice-ni.gov.uk/sites/default/files/publications/ofmdfm_dev/togetherbuilding-a-united-community-strategy.pdf (11. Juni 2020)

Feeney, Brian. The Troubles. Dublin: O’Brian Press, 2004.

Feuerring [Zinnar al-Nar]. Reg. Bahij Hojeij. On Line Films, 2003.

Gottdiener, Mark. „Culture, Ideology, and the Sign of the City“. The City and the Sign. An Introduction to Urban Semiotics. Hg. Mark Gottdiener und Alexandors Lagopoulos. New York: Columbia University Press, 1986. 202-218.

Grenzenloser Haß [Nothing Personal]. Reg. Thaddeaus O'Sullivan. Channel Four Films, 1995. Hanna, Brian. „Belfast: ,A Partnership Approach to Local Governance““. City Visions. Imagining Place, Enfranchising People. Hg. Frank Gaffkin und Mike Morrisey. London: Pluto Press, 1999.

Heatly, Colm. Interface. Flashpoints in Northern Ireland. Belfast: Lagan Books, 2004. Hill, John. Cinema and Northern Ireland: Film Culture and Politics. London: BFI, 2006. 
Belfast Interface Project. Interface Barriers, Peacelines and Defensive Architecture. 2017. https://www.belfastinterfaceproject.org/sites/default/files/publications/Interfaces $\%$ 20PDF.pdf (18. Mai 2021).

Im Schatten der Stadt [Tayf al-Madina]. Reg. Jean Chamoun. Nour Productions, 2000.

Intervention écrite de la sûreté générale. https://www.lorientlejour.com/article/615846/ article.html (21. Juni 2020).

Johnston, Kevin. In the Shadows of Giants. Dublin: Gill and Macmillan, 2008.

Kassir, Samir. Histoire de Beyrouth. Paris: Perrin, 2003.

Kennedy, Liam. Race and Urban Space in Contemporary American Literature. Edinburgh: University Press, 2003.

Khatib, Lina. Lebanese Cinema: Imagining Civil War and Beyond. London: Tauris, 2008. Llewllyn, Tim. Spirit of the Phoenix. Beirut and the Story of Lebanon. London: Tauris, 2010. Lynch, Kevin. The Image of the City. Cambridge, Massachusetts: M.I.T. Press, 1960.

McKittrick, David und McVea, David. Making Sense of the Troubles. London: Penguin, 2001. McNamee, Eoin. Resurrection Man, Production Notes. Dublin: Irish Film Institute Library, 1998. Melaugh, Martin: „Some Frequent Asked Questions. The Northern Irish Conflict“. CAIN. Conflict and Politics in Northern Ireland. https://cain.ulster.ac.uk/faq/faq2.htm (11 Juni 2020).

Mermier, Franck. Récits des villes: d'Aden à Beyrouth. Paris: Actes Sud, 2015.

Mitchell, Claire. Religion, Identity and Politics in Northern Ireland. Boundaries of Belonging and Belief. Aldershot: Ashgate, 2006.

Naeff, Judith. Precarious Imaginaries of Beirut. Basingstoke: Palgrave Macmillan, 2018.

O'Reilly, Camille. „The Irish Language as Symbol“. Symbols in Northern Ireland. Hg. Anthony Buckley. Belfast: The Institute of Irish Studies, 1998. 43-62.

Resurrection Man. Reg. Marc Evans. Revolution Films, 1998.

Rolstone, Bill. „Mothers, Whores and Villains: Images of Women in Novels of the Northern Ireland Conflict“. Race \& Class 31.1 (1989): 41-57.

Ross, David. Ireland. History of a Nation. Belfast: Lagan, 2005.

Smith, Gerry. The Novel and the Nation. Belfast: Pluto Press, 1997.

Smithey, Lee A. „Conflict Transformation, Cultural Innovation, and Loyalist Identity in Northern Ireland“. Culture and Belonging in Divided Societies. Contestation and Symbolic Landscapes. Hg. Marc Ross. Philadelphia: University of Pennsylvania Press, 2009. 85-106.

„Visualising the Conflict“. CAIN. Conflict and Politics in Northern Ireland. https://cain. ulster.ac. uk/victims/gis/maps/l-jpg/CAIN-Map_Belfast_Religion_Peacelines.jpg, (11. Juni 2020).

Welch, Robert. Concise Companion to Irish Literature. Oxford: OUP, 2000. 